\title{
SMALL CELL UNDIFFERENTIATED (NEUROENDOCRINE) TUMOR - MAXILLARY SINUS.
}

C. Bharath, Vijayalaxmi Rampure

1. Professor \& HOD, Department of Pathology, Vijayanagara Institute of Medical Sciences, Bellary.

2. Assistant Professor, Department of Pathology, Mahadevappa Rampure Medical College,Gulbarga.

\section{CORRESPONDING AUTHOR}

Dr. C. Bharath, Professor \& HOD,

Dept. of Pathology,

Vijayanagara Institute of Medical Sciences, Bellary.

E-mail: bhar5anu@yahoo.co.in

Ph: 00919483980702

ABSTRACT: BACKGROUND: Small cell undifferentiated (neuroendocrine) carcinomas in head and neck region is extremely uncommon neoplasm. These belong to the group of other blue cell tumors. CASE REPORT: An 11 year's girl presented with swelling in right cheek since 3 months, watering of right eyesince 2 months \& bleeding since 1 month. Examination revealed soft to firm mass in inferior meatus and was bleeding on touch. CT scan showed heterogeneous density in maxillary antrum. Biopsy was done. Histopathologically, diagnosis of Small cell carcinomaundifferentiated (neuroendocrine) type of maxillary sinus was made. CONCLUSION: These neoplasms of the nasal and paranasal sinuses are locally aggressive and have propensity for multiple local recurrences, and thus, early diagnosis and local control may be important for improved prognosis.

KEY WORDS: Paranasal sinuses, maxilla, neoplasm, small cell undifferentiated, neuroendocrine.

INTRODUCTION: Small cell undifferentiated (neuroendocrine) carcinoma in the head and neck region arises primarily from salivary glands, mainly parotid as well as in larynx, but rarely/uncommonly in the sino-nasal region ${ }^{3}$. The common neoplasms in the sino-nasal tract are squamous cell carcinoma, followed by Adenocarcinoma which is seen in older age, associated with history of smoking and chronic sinusitis. This case is presented because of its rarity in the younger age.

CASE REPORT: A young female child aged 11 years, presented with swelling in right side of cheek, since 03 months. Watering of right eye - 02 months and bleeding from right nose since 01 month. On examination of the right nose- there was a soft to firm red swelling in the inferior meatus, pushing inferior turbinate medially \& superiorly and was bleeding on touch and clinically diagnosed as Carcinoma of right maxilla. CT scan revealed a heterogenous density, soft tissue attenuation with right maxillary antrum as its epicenter and causing focal destruction of walls, suggesting malignant neoplasm - lymphoma / Rhabdomyosarcoma. Other investigations were within normal limits except for mild anemia.

PATHOLOGIC FINDINGS: Multiple grey white \& grey brown bits of irregular tissues, largest measuring $4^{*} 2^{*} 0.7 \mathrm{cms}$ in size with bony spicules were received. The surface showed papillae 
like processes. Bits were given, processed and stained with routine hematoxylin \& eosin. Histologically, showed small round to oval cells, having hyperchromatic nucleus with scant cytoplasm (Fig.1). These tumor cells were arranged in sheets, ribbons, nests and also in gland like pattern. Focal areas showed spindle like cells \& hemorrhagic spaces. These tumor cells also showed infiltration to the bony spicules \&stroma. A diagnosis of Small cell undifferentiated carcinoma- Right Maxillary sinus was made.

DISCUSSION: Small cell undifferentiated (neuroendocrine) carcinomas are extremely uncommon neoplasms in the sino-nasal tract. The median age of involvement for paranasal sinuses is 50 years, with equal gender distribution ${ }^{1}$. In our case, this neoplasm presented in young female child of 11 years of age. These tumors primarily arise in major salivary glands ${ }^{3}$, commonly the parotid, as well as larynx, but rarely occur in the sino-nasal region and hard palate.

Small cell undifferentiated carcinomas are aggressive with high potential for local recurrence $2 \& 6$. The occurrence of distant spread of primaries from sino-nasal region appears to be less frequent when compared to the small cell carcinoma of larynx or lung. Also these tumors have not been reported in association with tobacco use, as in contrast with laryngeal small cell carcinomas ${ }^{1}$.

In 1965, the first case of Small cell undifferentiated (neuroendocrine) carcinoma in the sino-nasal tract was described by Raychowdari. The most common neoplasmsin thesino-nasal regions are Squamous cell carcinoma and less commonly Adenocarcinoma \& adenoid cystic carcinoma. These tumors may produce ectopic hormones, but clinical manifestation of hormone production is uncommon. They have been described as an endocrine tumor of the Amine Precursor uptake of Decarboxylation system of Pearse ${ }^{4}$.

Histologically, showed spectrum of cell types, which grow as sheets, nests/ ribbons with areas of glandular pattern. The tumor cells are small, having round to oval nucleus with minimal cytoplasm and dispersed chromatin. Some of them were larger, with coarse chromatin and occasional small nucleoli and scant cytoplasm. Also sheets of spindle shaped cells are noticed in fibro-vascular stroma.

There is propensity for multiple recurrences with direct extension into the cranial bone and brain, which should be kept in mind ${ }^{1}$. The clinical behavior of these carcinomas is not well known because of its rarity; however, it seems more aggressive than the olfactory neuroblastoma 5 .

CONCLUSION: Small cell undifferentiated (neuroendocrine) carcinomas in the nasal cavity and paranasal sinuses are extremely uncommon aggressive neoplasm and has propensity for local recurrence and direct extension into the cranial bone and brain. Hence, early diagnosis along with histopathological examination and local control is important for improved prognosis. This type of neoplasm show poor response to neoadjuvent chemotherapy, unlike small cell carcinoma primary to other sites like lung.

\section{REFERENCES:}

1. Richard J Zarlo, Frank X Torses\& Jose Gomez: Nasal cavity \&Paranasal sinuses. Head \&NeckSurgical Pathology, Benz Z Pilch, Lippincott. 2001; 129-132.

2. Rajowski JE, Campanella RS \& Block LJ: Small cell carcinoma of the Paranasal sinuses. Otolaryngol Head Neck Surg. 1982; 90:516-577. 
3. Koss LG, Spiro RH, HajdeS: Small cell carcinoma of minor salivary gland origin. Cancer. 1972; 80: 737- 741.

4. Gould VE \&DeLellisRA: The neuroendocrine cell system, its tumors, hyperplasia and dysplasiaa. Principles \& Practice of Surgical Pathology,Vol-II,(editor-SG Silverberg), John Wallis, New York. 1983; 1487-1501.

5. HB Helliquist: Pathology of Nose \&Paranasal sinuses, Butter worths. 1990; 102-103.

6. Weiss MD, DeFries HO, Toxy JB, et al: Primary Small cell carcinomas of the Paranasal sinuses. Archives of Otolaryngol. 1983; 109:341-343.

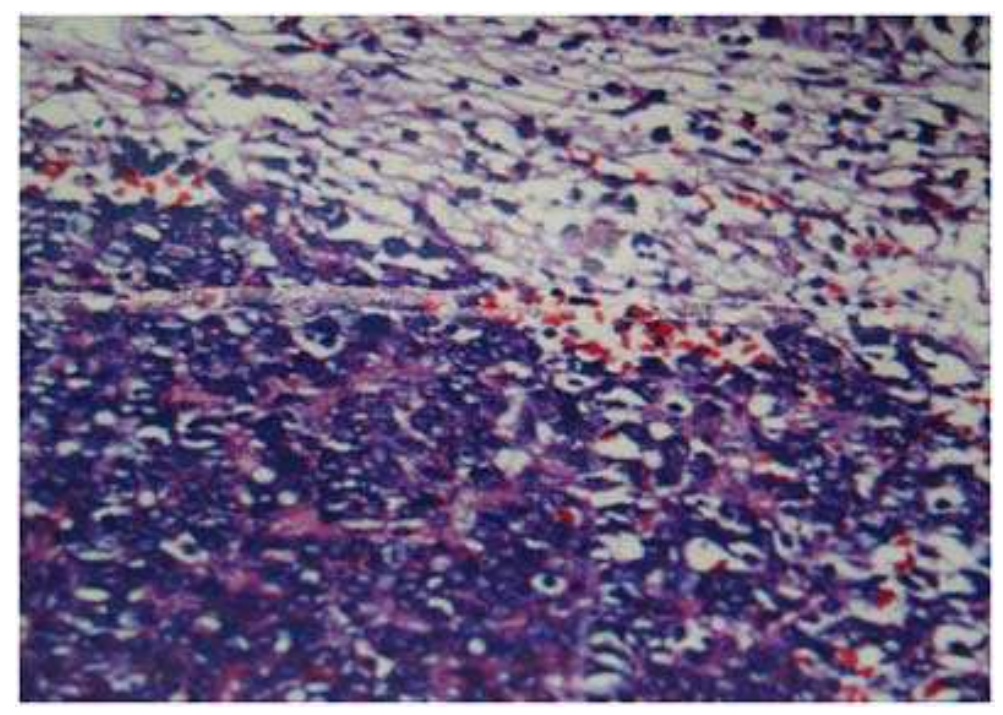

Fig.1. Histologically showing heterogenous cell, with hyperchromatic nucleus in cord and sheets. (H \& E. 45X) 